\title{
Magnetic order and ballistic spin transport in a sine-Gordon spin chain
}

\author{
B. M. Huddart $\odot,{ }^{1}$ M. Gomilšek $\odot,{ }^{2,1}$ T. J. Hicken $\odot,{ }^{1}$ F. L. Pratt,${ }^{3}$ S. J. Blundell $\odot,{ }^{4}$ P. A. Goddard $\odot,{ }^{5}$ S. J. Kaech, ${ }^{6}$ \\ J. L. Manson, ${ }^{6}$ and T. Lancaster (i) ${ }^{1}$ \\ ${ }^{1}$ Centre for Materials Physics, Durham University, Durham DH1 3LE, United Kingdom \\ ${ }^{2}$ Jožef Stefan Institute, Jamova c. 39, SI-1000 Ljubljana, Slovenia \\ ${ }^{3}$ ISIS Facility, STFC Rutherford Appleton Laboratory, Didcot OX11 OQX, United Kingdom \\ ${ }^{4}$ Department of Physics, Clarendon Laboratory, Oxford University, Parks Road, Oxford OX1 3PU, United Kingdom \\ ${ }^{5}$ Department of Physics, University of Warwick, Coventry CV4 7AL, United Kingdom \\ ${ }^{6}$ Department of Chemistry and Biochemistry, Eastern Washington University, Cheney, Washington 99004, USA
}

(Received 24 June 2020; revised 20 January 2021; accepted 25 January 2021; published 15 February 2021)

\begin{abstract}
We report the results of muon-spin spectroscopy $\left(\mu^{+} \mathrm{SR}\right)$ measurements on the staggered molecular spin chain [pym- $\mathrm{Cu}\left(\mathrm{NO}_{3}\right)_{2}\left(\mathrm{H}_{2} \mathrm{O}\right)_{2}$ ] (pym = pyrimidine), a material previously described using sine-Gordon field theory. Zero-field $\mu^{+} \mathrm{SR}$ reveals a long range magnetically ordered ground state below a transition temperature $T_{\mathrm{N}}=0.23(1) \mathrm{K}$. Using longitudinal-field (LF) $\mu^{+} \mathrm{SR}$ we investigate the dynamic response in applied magnetic fields $0<B<500 \mathrm{mT}$ and find evidence for ballistic spin transport. Our LF $\mu^{+}$SR measurements on the chiral spin chain $\left[\mathrm{Cu}(\mathrm{pym})\left(\mathrm{H}_{2} \mathrm{O}\right)_{4}\right] \mathrm{SiF}_{6} \cdot \mathrm{H}_{2} \mathrm{O}$ instead demonstrate one-dimensional spin diffusion, and the distinct spin transport in these two systems suggests that additional anisotropic interactions play an important role in determining the nature of spin transport in $S=1 / 2$ antiferromagnetic chains.
\end{abstract}

DOI: 10.1103/PhysRevB.103.L060405

At low temperatures, the one-dimensional (1D) antiferromagnetic (AF) spin chain hosts a range of exotic magnetic phenomena including quantum-critical fluctuations, emergent energy gaps and topological excitations. The ideal $S=1 / 2$ AF Heisenberg chain has a gapless excitation spectrum, but is highly sensitive to small modifications. A particularly dramatic effect results from the spins enjoying an alternating local environment, which is achieved in a so-called staggered spin chain [1]. This system hosts a magnetic-field-induced gapped phase described by sine-Gordon (SG) quantum-field theory, which predicts a complex excitation spectrum including solitons, antisolitons, and soliton-antisoliton bound states, known as breathers. Generalized hydrodynamic approaches show that transport in the SG field theory is ballistic, except in certain limits (including low temperature), where the semiclassical result predicting either ballistic or diffusive transport is recovered [2]. However, owing to the scarcity of model material systems, the nature of the spin transport has not been established in experimental realizations of this model. In this Letter, we investigate the staggered spin chain material [pym$\left.\mathrm{Cu}\left(\mathrm{NO}_{3}\right)_{2}\left(\mathrm{H}_{2} \mathrm{O}\right)_{2}\right]\left(\right.$ pym $=$ pyrimidine $\left.=\mathrm{C}_{4} \mathrm{H}_{4} \mathrm{~N}_{2}\right)$, hereafter $\mathrm{Cu}-\mathrm{PM}$, using muon-spin spectroscopy $\left(\mu^{+} \mathrm{SR}\right) . \mathrm{Cu}-\mathrm{PM}$ is one of relatively few experimental realizations of a staggered spin chain that is well described by SG theory $[3,4]$ and here we reveal the material's ground state and the character of its spin transport.

In $\mathrm{Cu}-\mathrm{PM}$, the $\mathrm{Cu}^{2+}$ ions form chains, with the primary magnetic exchange $(J=36.3(5) \mathrm{K}[5])$ being mediated by linking pym ligands [6]. It has been shown theoretically [7] that the material's staggered local $g$ tensor of neighboring $\mathrm{Cu}^{2+}$ ions produces an effective staggered internal magnetic field transverse but proportional in magnitude to the applied magnetic field. This internal field, which can also be produced by alternating Dzyaloshinskii-Moriya (DM) interactions along the chain, results in a magnetic-field-induced gap $\Delta$, which has been experimentally observed for $\mathrm{Cu}-\mathrm{PM}$ [4,5]. In addition, signatures of the three lowest breathers and a soliton predicted by SG theory have been observed with electron-spin resonance (ESR) [8]. At temperatures above the spin gap, in the perturbative spinon regime $\Delta<T<$ $J$, the soliton-breather superstructure is suppressed and the anisotropic contribution to the Hamiltonian due to the staggered $g$ tensors and DM interactions can be treated as a small perturbation to an underlying $S=1 / 2$ AF Heisenberg chain Hamiltonian with fractionalized spin- $1 / 2$ excitations called spinons [1]. This perturbation is predicted to result in a field shift and broadening of the ESR line shape [9], and the measured ESR parameters for Cu-PM show excellent quantitative agreement with these predictions [10].

While the subject of zero-field (ZF) magnetic order has been investigated in other SG spin chains, the magnetic ground state of $\mathrm{Cu}-\mathrm{PM}$ has not yet been established. Despite earlier claims that anomalies in the temperature dependence of the ESR frequency [11] and magnetic susceptibility [3] of Cu-benzoate $\left[\mathrm{Cu}\left(\mathrm{C}_{6} \mathrm{D}_{5} \mathrm{OO}\right)_{2} \cdot 3 \mathrm{D}_{2} \mathrm{O}\right]$ at $T \approx 0.8 \mathrm{~K}$ were due to an AF phase transition, no evidence for Néel ordering was found from neutron scattering experiments [12], and it was later argued that the ESR peak instead corresponds to an SG breather excitation [9]. A subsequent $\mu^{+}$SR study found no evidence of long-range order (LRO) down to $20 \mathrm{mK}$ [13]. On the other hand, $\mathrm{CuCl}_{2} \cdot 2 \mathrm{DMSO}$ (CDC) has been shown to acquire zero-field LRO below $T_{\mathrm{N}}=0.93 \mathrm{~K}$ [14]. The interchain 

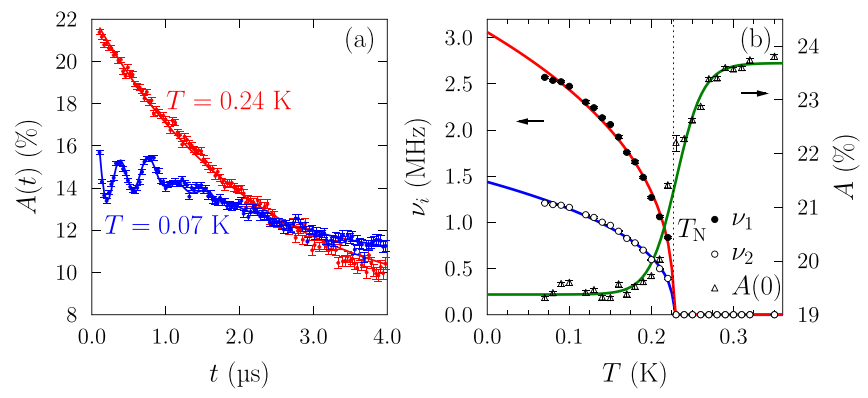

FIG. 1. (a) Example ZF spectra above and below the magnetic ordering temperature of Cu-PM. (b) Temperature dependence of the (left axis) precession frequencies and (right axis) initial asymmetry.

interactions responsible for this LRO lead to significant deviations from the SG model predictions below the $B_{\mathrm{c}}=3.9 \mathrm{~T}$ critical field for Néel order [15]. Muons are often sensitive to LRO in quasi-1D systems [16-18], which can be very difficult to detect using thermodynamic probes. In this study, we use $\mu^{+} \mathrm{SR}$ to show that the ground state of $\mathrm{Cu}-\mathrm{PM}$ is long-range ordered at temperatures below $T_{\mathrm{N}}=0.23(1) \mathrm{K}$. We determine muon stopping sites using density functional theory (DFT) and use these to provide further insight into the nature of the ordered moments. We also use $\mu^{+}$SR to explore spin transport above $T_{\mathrm{N}}$ in the perturbative spinon regime and show that it is ballistic at all measured temperatures, and contrast these results with measurements on the chiral staggered spin chain $\left[\mathrm{Cu}(\right.$ pym $\left.)\left(\mathrm{H}_{2} \mathrm{O}\right)_{4}\right] \mathrm{SiF}_{6} \cdot \mathrm{H}_{2} \mathrm{O}$, which instead demonstrate diffusive spin transport.

ZF $\mu^{+}$SR measurements $[19,20]$ reveal a state of LRO in $\mathrm{Cu}$-PM below $T_{\mathrm{N}}=0.23(1) \mathrm{K}$, indicated by the appearance of spontaneous oscillations at two frequencies [see Fig. 1(a)]. The spectra for $T<0.23 \mathrm{~K}$ were therefore fitted to $A(t)=\sum_{i=1}^{2} A_{i} e^{-\lambda_{i} t} \cos \left(2 \pi v_{i} t\right)+A_{3} e^{-\lambda_{3} t}+$ $A_{4} e^{-\sigma^{2} t^{2}}+A_{\mathrm{bg}}$, where the components with amplitudes $A_{1}$ and $A_{2}$ account for muons stopping in the sample at two magnetically distinct sites and undergoing coherent spin precession with frequencies $v_{i}$ and relaxation rates $\lambda_{i}$. Muons with their spins aligned parallel to the local field are relaxed only by dynamics and give rise to a purely relaxing component $A_{3}$, while those stopping outside of the sample result in a constant background with amplitude $A_{\text {bg }}$. We also find an additional component $A_{4}$ with Gaussian relaxation that is absent above $T_{\mathrm{N}}$, but has a relaxation rate $\sigma$ that is approximately constant below $T_{\mathrm{N}}$, with an average value of $0.41 \mu \mathrm{s}^{-1}$, and we discuss the possible origin of this relaxation in the Supplemental Material (page 2, paragraph 4) [20]. The amplitudes $A_{1}, A_{2}, A_{3}$ and $A_{4}$ reflect the fraction of muons in each distinct magnetic environment and these account for approximately $22 \%, 15 \%, 36 \%$, and $27 \%$ of the relaxing part of the asymmetry, respectively. The frequencies $v_{1}$ and $v_{2}$ were found to vary in fixed proportion, so we fixed $v_{2}=0.49 v_{1}$ in the fitting procedure. The frequencies $v_{i}(T)$, shown in Fig. 1(b), are proportional to the magnetic field at the muon site and act as an order parameter for the system. A fit to the critical scaling function $v_{1}(T)=v_{1}(0)\left(1-T / T_{\mathrm{N}}\right)^{\beta}$, appropriate near a second-order phase transition, yields an estimate $T_{\mathrm{N}}=0.23(1) \mathrm{K}$ for the ordering temperature and $\beta=0.39(1)$ for the critical exponent. The critical exponent $\beta$ obtained here is close to the value 0.367 of the 3D Heisenberg universality class [21], suggesting that the transition to $\mathrm{LRO}$ in $\mathrm{Cu}-\mathrm{PM}$ is three dimensional in nature. The precession frequencies $v_{1}(0)=3.1(1) \mathrm{MHz}$ and $v_{2}(0)=1.52(5) \mathrm{MHz}$ correspond to local magnetic fields of magnitudes $B_{1}(0)=$ 22.9(7) $\mathrm{mT}$ and $B_{2}(0)=11.2(3) \mathrm{mT}$, respectively, for each of the magnetically distinct muon stopping sites. Above $T_{\mathrm{N}}$, the spectra can be described by the sum of an exponential relaxation due to fluctuating disordered electronic moments and a constant background. The temperature dependence of the initial asymmetry is shown in Fig. 1(b) and exhibits a narrow transition between two approximately constant values, with a fit to an empirical logistic function yielding the same transition temperature as estimated above. After we account for the fraction of the asymmetry that is rapidly depolarized, we find that the amplitude $A_{3}$ corresponds to 0.32 of the asymmetry due to muons at sites that give rise to oscillations, consistent with what one would expect for a " $1 / 3$ tail." This suggests that the Gaussian relaxation below $T_{\mathrm{N}}$ is due to muons at a third magnetically distinct site, with the form of the relaxation pointing to a distribution of positions, such that the muons experience a range of local fields.

To quantify the ordered moments and any structural distortions due to the presence of a muon, we have carried out density functional theory (DFT) calculations of the muon stopping sites [20] using the plane-wave basis-set electronic structure code CASTEP [22]. We identify three distinct classes of muon stopping site and show these in Fig. 2. Sites where the muon sits around $1 \AA$ from an $\mathrm{O}$ atom in a nitrate group [Fig. 2(a)] or $\mathrm{H}_{2} \mathrm{O}$ ligand [Fig. 2(b)] are the lowest and second lowest energy classes of sites, respectively. We also find candidate sites where the muon sits $1.0 \AA$ from an $\mathrm{N}$ atom in a pym ligand [Fig. 2(c)], which are substantially higher in energy and result in larger local distortions to the crystal structure. These sites can be mapped to features in the ZF spectra by considering the dipolar fields resulting from candidate AF magnetic structures. From our dipolar field calculations [20] we obtain fields of $9-40 \mathrm{mT} / \mu_{\mathrm{Cu}}$ for the nitrate site, $57-63 \mathrm{mT} / \mu_{\mathrm{Cu}}$ for the $\mathrm{H}_{2} \mathrm{O}$ site, and $93-99 \mathrm{mT} / \mu_{\mathrm{Cu}}$ for the $\mathrm{N}(\mathrm{pym})$ site, where $\mu_{\mathrm{Cu}}$ is the ordered moment of the $\mathrm{Cu}^{2+}$ ions in Bohr magnetons. We note that the relative size of the calculated fields for the $\mathrm{H}_{2} \mathrm{O}$ and nitrate sites is consistent with the ratio between frequencies $v_{1}$ and $v_{2}$, respectively. This assignment gives an estimate $\mu_{\mathrm{Cu}} \approx 0.38 \mu_{\mathrm{B}}$ for the ordered moment. This moment size would give a precession frequency of around $5 \mathrm{MHz}$ for the $\mathrm{N}(\mathrm{pym})$ site, which should be resolvable. However, it is possible that sites of this type (or a similar set of sites close to the aromatic ring) could instead experience a distribution of static fields due to the magnetic structure and thus be responsible for the Gaussian relaxation seen below $T_{\mathrm{N}}$.

Although the ideal 1D $S=1 / 2$ quantum Heisenberg AF is not expected to show long-range ordering for $T>0$ [23], experimental realizations of this model are found to order at low, but nonzero temperature due to the presence of interactions between chains. A useful figure of merit for the degree to which low dimensionality is achieved is the ratio $T_{\mathrm{N}} / J$, as this quantity should be zero for the ideal case and close to unity for an isotropic material. This quantity can also be used to estimate the interchain coupling $J^{\prime}$ using a formula obtained from 

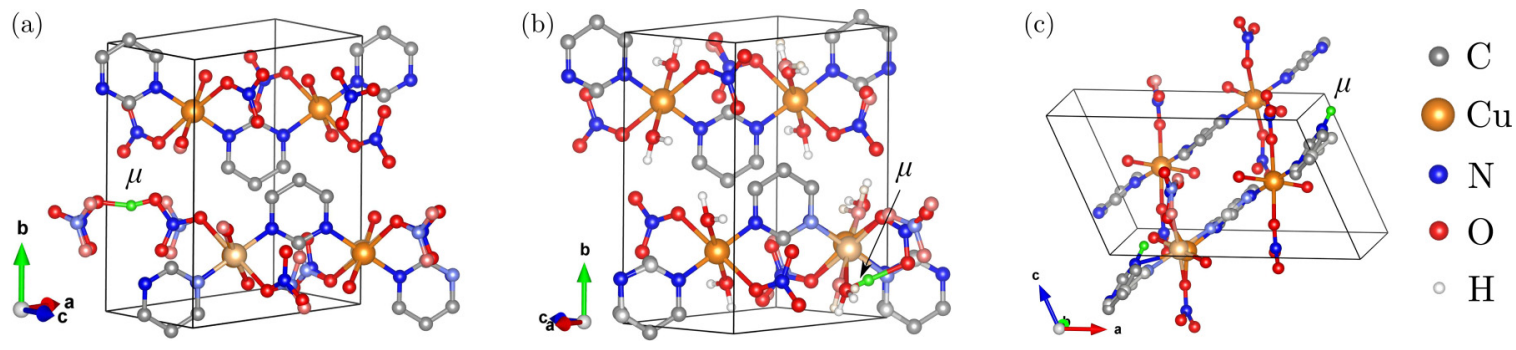

FIG. 2. Low-energy muon sites in Cu-PM. Lighter spheres represent the ionic positions in the unit cell without the muon. H atoms have been omitted for clarity where appropriate. (a) The nitrate site. (b) $\mathrm{The}_{2} \mathrm{O}$ site. (c) the $\mathrm{N}(\mathrm{pym})$ site.

the results of quantum Monte Carlo calculations on $\mathrm{AF}$ chains [24]. For Cu-PM we obtain $T_{\mathrm{N}} / J=6.3 \times 10^{-3}, J^{\prime} \approx 0.09 \mathrm{~K}$, and $\left|J^{\prime} / J\right|=2.6 \times 10^{-3}$, though we note that additional terms present in the Hamiltonian for a staggered spin chain are likely to lower $T_{\mathrm{N}}$ below the value expected for a simple 1D AF chain and therefore this estimate of $J^{\prime}$ serves as a lower bound. We can also use these parameters to estimate the size of the ordered moment, using the formula $m \approx 2.034\left|J^{\prime} / J\right|^{1 / 2}$ obtained from a model of weakly coupled AF spin chains [25]. For Cu-PM this yields $m \approx 0.1 \mu_{\mathrm{B}}$, demonstrating that the ordered moment is heavily renormalized due to enhanced quantum fluctuations in this low-dimensional system, an effect that is also seen, to a lesser extent, in our estimate $\mu_{\mathrm{Cu}} \approx$ $0.38 \mu_{\mathrm{B}}$ obtained by considering the dipolar field at the muon sites.

The values of $T_{\mathrm{N}} / J$ (Table I) suggest that the degree of isolation of the $\mathrm{Cu}^{2+}$ chains in Cu-PM is similar to that found in the well-isolated $1 \mathrm{D} \mathrm{AF}$ linear spin chain copper pyrazine dinitrate $\left[\mathrm{Cu}(\mathrm{pyz})\left(\mathrm{NO}_{3}\right)_{2}\right]$ [16]. In $\mathrm{CDC}$, which, like $\mathrm{Cu}-\mathrm{PM}$, exhibits alternating $g$ tensors and DM interactions, the ground state in zero field is a collinear AF below $T_{\mathrm{N}}=0.93 \mathrm{~K}$, with a moment $0.44(5) \mu_{\mathrm{B}}$ along an Ising-like easy axis [14]. The higher transition temperature in CDC may be due to the lower DM energy in this system [14], which is an order of magnitude smaller relative to $J$ than the values established for $\mathrm{Cu}-\mathrm{PM}$ [4] and Cu-benzoate [7]. A more recent example of a staggered chain is the chiral chain $\left[\mathrm{Cu}(\mathrm{pym})\left(\mathrm{H}_{2} \mathrm{O}\right)_{4}\right] \mathrm{SiF}_{6} \cdot \mathrm{H}_{2} \mathrm{O}$, in which the environments of adjacent $\mathrm{Cu}^{2+}$ ions are related by $4_{1}$ screw symmetry [28]. At zero field, its magnetism is well described as a $1 \mathrm{D} S=1 / 2$ Heisenberg AF, with intrachain exchange $J=42(1) \mathrm{K}$ and no magnetic order detected down to $20 \mathrm{mK}$ [27]. The suppression of LRO in this system may be due to a uniform DM interaction that alternates in sign from chain to chain, which has been shown theoretically

TABLE I. Ordering temperature $T_{\mathrm{N}}$ and intrachain exchange $J$ for a series of $\mathrm{Cu}$-chain compounds.

\begin{tabular}{lccc}
\hline \hline & $T_{\mathrm{N}}(\mathrm{K})$ & $J(\mathrm{~K})$ & $T_{\mathrm{N}} / J$ \\
\hline $\mathrm{Cu}($ pyz $)\left(\mathrm{NO}_{3}\right)_{2}[16,26]$ & $0.105(2)$ & $10.3(1)$ & $1.0 \times 10^{-2}$ \\
$\mathrm{Cu}$-benzoate [3,13] & $<0.02$ & $18.2(1)$ & $<10^{-3}$ \\
$\mathrm{CDC}[14]$ & 0.93 & $16.9(1)$ & $5.5 \times 10^{-2}$ \\
{$\left[\mathrm{Cu}(\right.$ pym $\left.)\left(\mathrm{H}_{2} \mathrm{O}\right)_{4}\right] \mathrm{SiF}_{6} \cdot \mathrm{H}_{2} \mathrm{O}[27]$} & $<0.02$ & $42(1)$ & $<5 \times 10^{-4}$ \\
$\mathrm{Cu}-\mathrm{PM}[4,5]$ & $0.23(1)$ & $36.3(5)$ & $6.3 \times 10^{-3}$ \\
\hline \hline
\end{tabular}

to effectively result in a cancellation of the interchain interaction [29].

Longitudinal-field (LF) $\mu^{+}$SR is often used to investigate low-energy dynamics in $1 \mathrm{D}$ materials, including spin chains [30] and ladders [31]. The principle of these measurements is to work at a temperature $T$ in the $1 \mathrm{D}$ regime, $T_{\mathrm{N}} \ll T \lesssim J$ (i.e., above any ordering temperature, and below the energy scale of the spin exchange along the chain), where collective low-dimensional behavior of the spins is expected. In cases where the isotropic hyperfine coupling $\mathcal{A}$ dominates over the dipolar coupling, the field dependence of the muon-spin relaxation rate $\lambda$ is given by $\lambda(B)=\left(\mathcal{A}^{2} / 4\right) f\left(\omega_{\mathrm{e}}\right)$, where $f(\omega)$ is the spectral density and the probe frequency $\omega_{\mathrm{e}}=\gamma_{\mathrm{e}} B$, where $\gamma_{\mathrm{e}}$ is the electron gyromagnetic ratio [32]. Field-dependent measurements [30,33] can be used to distinguish between the two main types of spin transport possible in a 1D chain, namely spin diffusion or ballistic transport, since their spin autocorrelation functions have different associated spectral densities: $f(\omega) \propto \omega^{-1 / 2}$ for diffusive transport and $f(\omega) \propto$ $\ln (c J / \omega)$ for ballistic motion, where $c$ is a constant of order unity. We have carried out LF $\mu^{+}$SR measurements on $\mathrm{Cu}-\mathrm{PM}$ to study the spin dynamics in this system. The data were fitted to

$$
A(t)=A_{\mathrm{rel}} G_{z}^{\mathrm{KT}}(\Lambda, B) e^{-\lambda t}+A_{\mathrm{bg}},
$$

which includes the contribution of the quasistatic nuclear moments through the LF Kubo-Toyabe function $G_{z}^{\mathrm{KT}}(\Lambda, B)$, where $\Lambda$ is the width of the resulting field distribution, and exponential relaxation due to the dynamics of the electronic spins, with relaxation rate $\lambda$. Muons stopping outside of the sample give rise to a constant background asymmetry with amplitude $A_{\mathrm{bg}}$.

The field dependence of the relaxation rate $\lambda$ is shown in Fig. 3. In Fig. 3(a) we show $\lambda(B)$ at $T=1.6 \mathrm{~K}$, alongside fits obtained from three different models. A fit to a function of the form $\lambda \propto B^{-n}$, appropriate for diffusive spin transport, yields $n=0.2$, which is much smaller than the theoretically predicted value of $n=0.5$ for one-dimensional diffusion. As seen in Fig. 3(a), a function of this form with $n=0.5$ does not describe the data. However, a function of the form $\lambda(B)=$ $a \ln (c J / B)$, appropriate for ballistic transport, provides a good fit to the data with $c=0.16$. In Fig. 3(b), we show the results of fitting the ballistic model to the data measured at several different temperatures; the model provides a good fit to the data at all of the measured temperatures and even up to $T \approx 2 J$. 

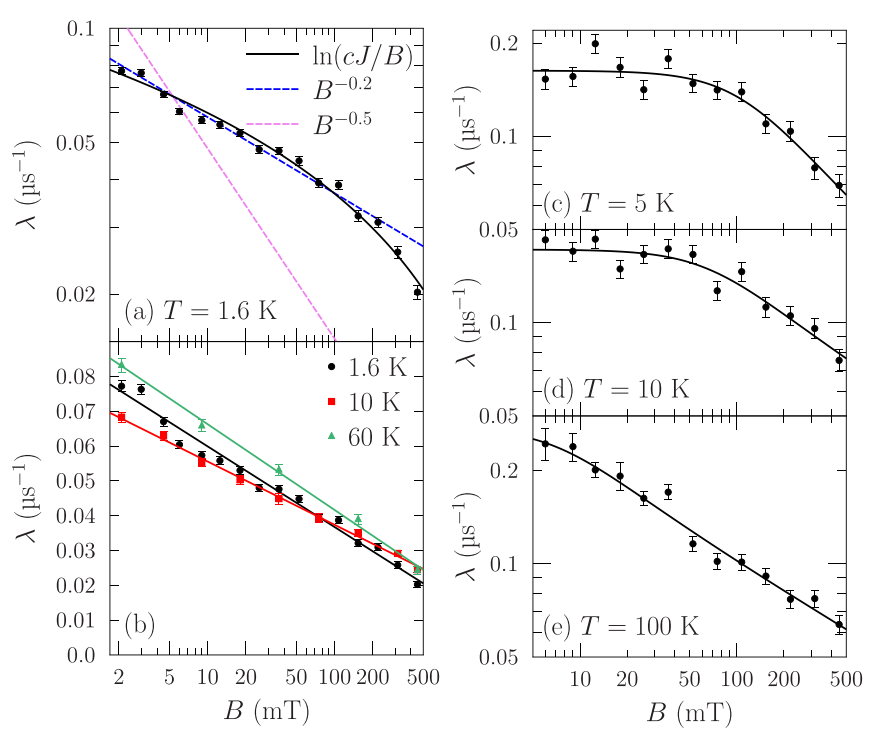

FIG. 3. (a) Field dependence of the LF relaxation rate in $\mathrm{Cu}-\mathrm{PM}$ at $T=1.6 \mathrm{~K}$ with fits appropriate for either diffusive or ballistic spin transport. (b) Field dependence of relaxation rate and fits describing ballistic spin transport. Field dependence of the LF relaxation rate for $\left[\mathrm{Cu}(\right.$ pym $\left.)\left(\mathrm{H}_{2} \mathrm{O}\right)_{4}\right] \mathrm{SiF}_{6} \cdot \mathrm{H}_{2} \mathrm{O}$ at (c) $T=5 \mathrm{~K}$, (d) $T=10 \mathrm{~K}$, and (e) $T=100 \mathrm{~K}$, with fits to Eq. (2).

A recent study [27] on the chiral staggered spin chain $\left[\mathrm{Cu}(\mathrm{pym})\left(\mathrm{H}_{2} \mathrm{O}\right)_{4}\right] \mathrm{SiF}_{6} \cdot \mathrm{H}_{2} \mathrm{O}$ found staggered $g$ tensors, a staggered susceptibility, and a spin gap that opens on the application of a magnetic field, all of which are reminiscent of phenomena observed in nonchiral staggered chains. We therefore carried out LF $\mu^{+} \mathrm{SR}$ measurements on this system to compare its spin dynamics with those measured for $\mathrm{Cu}-\mathrm{PM}$. For $B>10 \mathrm{mT}$ the spectra are well described by exponential relaxation and we show the field-dependent relaxation rates $\lambda$ in Figs. 3(c)-3(e) (details on the fitting at lower fields can be found in the Supplemental Material [20]). As seen in Fig. 3(c), the relaxation rate $\lambda(B)$ is approximately constant for fields up to around $50 \mathrm{mT}$ at $T=5 \mathrm{~K}$. We account for this behavior using a model for anisotropic spin diffusion, where the spectral density has the form

$$
f(\omega)=\frac{1}{\sqrt{2 D_{\|} D_{\perp}}}\left(\frac{1+\sqrt{1+\left(\omega / 2 D_{\perp}\right)^{2}}}{2\left[1+\left(\omega / 2 D_{\perp}\right)^{2}\right]}\right)^{n},
$$

with $D_{\|}$and $D_{\perp}$ being the intrachain and interchain diffusion rates, respectively [34]. This function shows a transition from a constant low frequency value to a power-law behavior $f(\omega) \propto \omega^{-n}$ at a crossover frequency proportional to $D_{\perp}$. [This model does not work well for Cu-PM, as there $\lambda(B)$ is not approximately constant at low fields.] Fitting the data measured at $5 \mathrm{~K}$ to this model, we obtain $D_{\perp}=10^{10} \mathrm{~s}^{-1}$ and $n=0.46(12)$. This value of $n$ is consistent with theory for diffusive transport $(n=0.5)$. As the temperature is raised we observe a shift in the crossover to lower fields [see Figs. 3(d) and 3(e)], corresponding to a decrease in $D_{\perp}$, and we also find that $D_{\|}$increases with increasing temperature.
We note that the fits to the data at $10 \mathrm{~K}$ [Fig. 3(d)] and $100 \mathrm{~K}$ [Fig. 3(e)] yield values of $n$ closer to 0.3, which, although noticeably smaller than theoretically predicted, are not too dissimilar from the value $n \approx 0.35$ measured for the linear chain DEOCC-TCNQF 4 [33]. Furthermore, we would expect the model in Eq. (2) to be most accurate at $T=5 \mathrm{~K}$ because, as the temperature approaches (or exceeds) $T \approx J$, the muons are responding not only to delocalized spin excitations but also to the quasi-independent spin flips introduced by thermal fluctuations.

Both ballistic $[35,36]$ and diffusive $[30,33,37]$ spin transport have previously been observed experimentally in $S=1 / 2$ AF spin chains, although the latter is far more common. Theoretically, the nature of spin transport in these systems remains controversial, with some studies claiming that the transport in these systems is necessarily ballistic [38-40], while other studies conclude that the transport is diffusive [41,42]. Furthermore, it has been suggested that, in the presence of a periodic lattice potential, diffusion can coexist with ballistic transport [43]. For the vast majority of the data measured for $\mathrm{Cu}-\mathrm{PM}$ we are within the perturbative spinon regime, where the soliton and breather modes are suppressed, leaving spinons as the excitations most likely responsible for the observed spin transport. The anisotropic term in the Hamiltonian due to the staggered $g$ tensor and DM interaction is likely to modify the spectral density of the spin excitations compared with those found in nonstaggered $S=1 / 2$ AF spin chains and could therefore be responsible for their distinct transport. There is evidence for other excitations, such as interbreather transitions [44], in this intermediate temperature regime, which could also influence the spin transport. The transport in $\left[\mathrm{Cu}(\mathrm{pym})\left(\mathrm{H}_{2} \mathrm{O}\right)_{4}\right] \mathrm{SiF}_{6} \cdot \mathrm{H}_{2} \mathrm{O}$ is very different, despite both systems being expected to occupy a similar regime of behavior (i.e., where the temperature exceeds the spin gap, but is smaller than the exchange strength $J$ ). However, we note that the size of the gap in $\left[\mathrm{Cu}(\mathrm{pym})\left(\mathrm{H}_{2} \mathrm{O}\right)_{4}\right] \mathrm{SiF}_{6} \cdot \mathrm{H}_{2} \mathrm{O}$ and its linear field dependence do not fit with the predictions of the SG model [27]. These differences have been attributed to additional interactions arising from the fourfold periodic chiral structure [27], and our results here suggest that these interactions also lead to spin transport distinct from that found in a traditional (nonchiral) staggered chain.

In conclusion, we have demonstrated the existence of longrange magnetic order in the staggered spin chain compound [pym- $\left.\mathrm{Cu}\left(\mathrm{NO}_{3}\right)_{2}\left(\mathrm{H}_{2} \mathrm{O}\right)_{2}\right]$ with $T_{\mathrm{N}}=0.23(1) \mathrm{K}$. Our LF $\mu^{+} \mathrm{SR}$ measurements show that the transport of the spin excitations detected by the muon is ballistic in the perturbative spinon regime, $\Delta<T<J$, whereas the transport in the chiral spin chain $\left[\mathrm{Cu}(\right.$ pym $\left.)\left(\mathrm{H}_{2} \mathrm{O}\right)_{4}\right] \mathrm{SiF}_{6} \cdot \mathrm{H}_{2} \mathrm{O}$ is shown to be diffusive. These results demonstrate the crucial role played by additional anisotropic terms in the Hamiltonian in determining the nature of the spin transport in $S=1 / 2 \mathrm{AF}$ spin chains. Establishing the sensitivity of the muon to the magnetic excitations in these systems paves the way for $\mu^{+} \mathrm{SR}$ measurements at higher magnetic fields, where implanted muons could provide insight into the soliton-breather regime at $T \ll \Delta$. 
Research data from this paper will be made available via Ref. [45].

Experiments were carried out at the STFC-ISIS Facility and we are grateful for the provision of beam time. We thank J. Singleton and J. L. Musfeldt for useful discussion. This work is supported by EPSRC (UK), under Grants No. EP/N024028/1 and No. EP/N032128/1. We acknowledge computing resources provided by Durham Hamilton HPC. M.G. acknowledges support by the Slovenian Research Agency under Project No. Z1-1852. Work at EWU was supported by the US National Science Foundation under Grant No. DMR-1703003. This project has received funding from the European Research Council (ERC) under the European Union's Horizon 2020 research and innovation program (Grant Agreement No. 681260).
[1] T. Giamarchi, Quantum Physics in One Dimension (Clarendon, Oxford, 2004).

[2] B. Bertini, L. Piroli, and M. Kormos, Transport in the sineGordon field theory: From generalized hydrodynamics to semiclassics, Phys. Rev. B 100, 035108 (2019).

[3] D. C. Dender, D. Davidović, D. H. Reich, C. Broholm, K. Lefmann, and G. Aeppli, Magnetic properties of a quasi-onedimensional $S=1 / 2$ antiferromagnet: Copper benzoate, Phys. Rev. B 53, 2583 (1996).

[4] R. Feyerherm, S. Abens, D. Günther, T. Ishida, M. Meißner, M. Meschke, T. Nogami, and M. Steiner, Magnetic-field induced gap and staggered susceptibility in the $S=1 / 2$ chain $\left[\mathrm{PM} \cdot \mathrm{Cu}\left(\mathrm{NO}_{3}\right)_{2} \cdot\left(\mathrm{H}_{2} \mathrm{O}\right)_{2}\right]_{n}(\mathrm{PM}=$ pyrimidine $)$, J. Phys. Condens. Matter 12, 8495 (2000).

[5] A. U. B. Wolter, H. Rakoto, M. Costes, A. Honecker, W. Brenig, A. Klümper, H.-H. Klauss, F. J. Litterst, R. Feyerherm, D. Jérome, and S. Süllow, High-field magnetization study of the $S=\frac{1}{2}$ antiferromagnetic Heisenberg chain $\left[\mathrm{PMCu}\left(\mathrm{NO}_{3}\right)_{2}\left(\mathrm{H}_{2} \mathrm{O}\right)_{2}\right]_{n}$ with a field-induced gap, Phys. Rev. B 68, 220406(R) (2003).

[6] M. Yasui, Y. Ishikawa, N. Akiyama, T. Ishida, T. Nogami, and F. Iwasaki, Dipyrimidine-copper(II) dinitrate complexes showing magnetic interactions, Acta Crystallogr. B 57, 288 (2001).

[7] M. Oshikawa and I. Affleck, Field-Induced Gap in $S=1 / 2$ Antiferromagnetic Chains, Phys. Rev. Lett. 79, 2883 (1997).

[8] S. A. Zvyagin, A. K. Kolezhuk, J. Krzystek, and R. Feyerherm, Excitation Hierarchy of the Quantum Sine-Gordon Spin Chain in a Strong Magnetic Field, Phys. Rev. Lett. 93, 027201 (2004).

[9] M. Oshikawa and I. Affleck, Low-Temperature Electron Spin Resonance Theory for Half-Integer Spin Antiferromagnetic Chains, Phys. Rev. Lett. 82, 5136 (1999).

[10] S. A. Zvyagin, A. K. Kolezhuk, J. Krzystek, and R. Feyerherm, Electron Spin Resonance in Sine-Gordon Spin Chains in the Perturbative Spinon Regime, Phys. Rev. Lett. 95, 017207 (2005).

[11] K. Oshima, K. Okuda, and M. Date, Antiferromagnetic resonance in copper benzoate below $1 \mathrm{~K}$, J. Phys. Soc. Jpn. 44, 757 (1978).

[12] D. C. Dender, P. R. Hammar, D. H. Reich, C. Broholm, and G. Aeppli, Direct Observation of Field-Induced Incommensurate Fluctuations in a One-Dimensional $S=1 / 2$ Antiferromagnet, Phys. Rev. Lett. 79, 1750 (1997).

[13] T. Asano, H. Nojiri, W. Higemoto, A. Koda, R. Kadono, and Y. Ajiro, $\mu$ SR study of $\mathrm{Cu}$ benzoate at very low temperatureexistence or nonexistence of long range order in coupled chains, J. Phys. Soc. Jpn 71, 594 (2002).

[14] Y. Chen, M. B. Stone, M. Kenzelmann, C. D. Batista, D. H. Reich, and C. Broholm, Phase diagram and spin Hamiltonian of weakly-coupled anisotropic $S=\frac{1}{2}$ chains in $\mathrm{CuCl}_{2}$. $2\left(\left(\mathrm{CD}_{3}\right)_{2} \mathrm{SO}\right)$, Phys. Rev. B 75, 214409 (2007).
[15] M. Kenzelmann, Y. Chen, C. Broholm, D. H. Reich, and Y. Qiu, Bound Spinons in an Antiferromagnetic $S=1 / 2$ Chain with a Staggered Field, Phys. Rev. Lett. 93, 017204 (2004).

[16] T. Lancaster, S. J. Blundell, M. L. Brooks, P. J. Baker, F. L. Pratt, J. L. Manson, C. P. Landee, and C. Baines, Magnetic order in the quasi-one-dimensional spin-1/2 molecular chain compound copper pyrazine dinitrate, Phys. Rev. B 73, 020410 (2006).

[17] P. A. Goddard, J. L. Manson, J. Singleton, I. Franke, T. Lancaster, A. J. Steele, S. J. Blundell, C. Baines, F. L. Pratt, R. D. McDonald, O. E. Ayala-Valenzuela, J. F. Corbey, H. I. Southerland, P. Sengupta, and J. A. Schlueter, Dimensionality Selection in a Molecule-Based Magnet, Phys. Rev. Lett. 108, 077208 (2012).

[18] T. Lancaster, P. A. Goddard, S. J. Blundell, F. R. Foronda, S. Ghannadzadeh, J. S. Möller, P. J. Baker, F. L. Pratt, C. Baines, L. Huang, J. Wosnitza, R. D. McDonald, K. A. Modic, J. Singleton, C. V. Topping, T. A. W. Beale, F. Xiao, J. A. Schlueter, A. M. Barton, R. D. Cabrera, K. E. Carreiro, H. E. Tran, and J. L. Manson, Controlling Magnetic Order and Quantum Disorder in Molecule-Based Magnets, Phys. Rev. Lett. 112, 207201 (2014).

[19] S. J. Blundell, Spin-polarized muons in condensed matter physics, Contemp. Phys. 40, 175 (1999).

[20] See Supplemental Material at http://link.aps.org/supplemental/ 10.1103/PhysRevB.103.L060405 for details of the $\mu^{+}$SR measurements and the density functional theory and dipolar field calculations, which includes Refs. [46-48].

[21] S. Blundell, Magnetism in Condensed Matter (Oxford University Press, Oxford, 2001).

[22] S. J. Clark, M. D. Segall, C. J. Pickard, P. J. Hasnip, M. I. J. Probert, K. Refson, and M. C. Payne, First principles methods using CASTEP, Z. Kristallogr. Cryst. Mater 220, 567 (2005).

[23] S. Sachdev, Quantum Phase Transitions, 2nd ed. (Cambridge University Press, Cambridge, 2011).

[24] C. Yasuda, S. Todo, K. Hukushima, F. Alet, M. Keller, M. Troyer, and H. Takayama, Néel Temperature of Quasi-LowDimensional Heisenberg Antiferromagnets, Phys. Rev. Lett. 94, 217201 (2005).

[25] H. J. Schulz, Dynamics of Coupled Quantum Spin Chains, Phys. Rev. Lett. 77, 2790 (1996).

[26] P. R. Hammar, M. B. Stone, D. H. Reich, C. Broholm, P. J. Gibson, M. M. Turnbull, C. P. Landee, and M. Oshikawa, Characterization of a quasi-one-dimensional spin-1/2 magnet which is gapless and paramagnetic for $g \mu_{B} H \lesssim J$ and $k_{B} T \ll J$, Phys. Rev. B 59, 1008 (1999).

[27] J. Liu, S. Kittaka, R. D. Johnson, T. Lancaster, J. Singleton, T. Sakakibara, Y. Kohama, J. van Tol, A. Ardavan, B. H. Williams, S. J. Blundell, Z. E. Manson, J. L. Manson, and P. A. Goddard, 
Unconventional Field-Induced Spin Gap in an $S=1 / 2$ Chiral Staggered Chain, Phys. Rev. Lett. 122, 057207 (2019).

[28] D. B. Cordes and R. D. Rogers, Enantiomorphic helical coordination polymers of $\left\{\left[\mathrm{M}(\text { pyrimidine })\left(\mathrm{OH}_{2}\right)_{4}\right]\left[\mathrm{SiF}_{6}\right] \mathrm{H}_{2} \mathrm{O}\right\}_{\infty}$ $\left(\mathrm{M}=\mathrm{Co}^{2+}, \mathrm{Cu}^{2+}, \mathrm{Zn}^{2+}\right)$, Cryst. Growth Des 7, 1943 (2007).

[29] W. Jin and O. A. Starykh, Phase diagram of weakly coupled Heisenberg spin chains subject to a uniform DzyaloshinskiiMoriya interaction, Phys. Rev. B 95, 214404 (2017).

[30] F. Xiao, J. S. Möller, T. Lancaster, R. C. Williams, F. L. Pratt, S. J. Blundell, D. Ceresoli, A. M. Barton, and J. L. Manson, Spin diffusion in the low-dimensional molecular quantum Heisenberg antiferromagnet $\mathrm{Cu}(\mathrm{pyz})\left(\mathrm{NO}_{3}\right)_{2}$ detected with implanted muons, Phys. Rev. B 91, 144417 (2015).

[31] T. Lancaster, F. Xiao, B. M. Huddart, R. C. Williams, F. L. Pratt, S. J. Blundell, S. J. Clark, R. Scheuermann, T. Goko, S. Ward, J. L. Manson, C. Rüegg, and K. W. Krämer, Quantum magnetism in molecular spin ladders probed with muon-spin spectroscopy, New J. Phys. 20, 103002 (2018).

[32] F. Devreux, J.-P. Boucher, and M. Nechtschein, Temps de relaxation nucléaire T1D et T1 $\rho$ en présence de mouvement de spins électroniques, J. Phys. France 35, 271 (1974).

[33] F. L. Pratt, S. J. Blundell, T. Lancaster, C. Baines, and S. Takagi, Low-Temperature Spin Diffusion in a Highly Ideal $S=\frac{1}{2}$ Heisenberg Antiferromagnetic Chain Studied by Muon Spin Relaxation, Phys. Rev. Lett. 96, 247203 (2006).

[34] K. Mizoguchi, Spin dynamics study in conducting polymers by magnetic resonance, Jpn. J. Appl. Phys. 34, 1 (1995).

[35] T. Lancaster, P. J. Baker, F. L. Pratt, S. J. Blundell, W. Hayes, and D. Prabhakaran, Persistent dynamics in the $S=$ $1 / 2$ quasi-one-dimensional chain compound $\mathrm{Rb}_{4} \mathrm{Cu}\left(\mathrm{MoO}_{4}\right)_{3}$ probed with muon-spin relaxation, Phys. Rev. B 85, 184404 (2012).

[36] H. Maeter, A. A. Zvyagin, H. Luetkens, G. Pascua, Z. Shermadini, R. Saint-Martin, A. Revcolevschi, C. Hess, B. Büchner, and H.-H. Klauss, Low temperature ballistic spin transport in the $S=1 / 2$ antiferromagnetic Heisenberg chain compound $\mathrm{SrCuO}_{2}$, J. Phys. Condens. Matter 25, 365601 (2013).
[37] K. R. Thurber, A. W. Hunt, T. Imai, and F. C. Chou, ${ }^{17}$ O NMR Study of $q=0$ Spin Excitations in a Nearly Ideal $S=\frac{1}{2} 1 \mathrm{D}$ Heisenberg Antiferromagnet, $\mathrm{Sr}_{2} \mathrm{CuO}_{3}$, up to $800 \mathrm{~K}$, Phys. Rev. Lett. 87, 247202 (2001).

[38] H. Castella, X. Zotos, and P. Prelovšek, Integrability and Ideal Conductance at Finite Temperatures, Phys. Rev. Lett. 74, 972 (1995).

[39] B. N. Narozhny, A. J. Millis, and N. Andrei, Transport in the XXZ model, Phys. Rev. B 58, R2921 (1998).

[40] J. Benz, T. Fukui, A. Klümper, and C. Scheeren, On the finite temperature Drude weight of the anisotropic Heisenberg chain, J. Phys. Soc. Jpn. 74, 181 (2005).

[41] X. Zotos, Finite Temperature Drude Weight of the OneDimensional Spin-1/2 Heisenberg model, Phys. Rev. Lett. 82, 1764 (1999).

[42] S. Grossjohann and W. Brenig, Hydrodynamic limit for the spin dynamics of the Heisenberg chain from quantum Monte Carlo calculations, Phys. Rev. B 81, 012404 (2010).

[43] J. Sirker, R. G. Pereira, and I. Affleck, Diffusion and Ballistic Transport in One-Dimensional Quantum Systems, Phys. Rev. Lett. 103, 216602 (2009).

[44] A. C. Tiegel, A. Honecker, T. Pruschke, A. Ponomaryov, S. A. Zvyagin, R. Feyerherm, and S. R. Manmana, Dynamical properties of the sine-Gordon quantum spin magnet $\mathrm{Cu}-\mathrm{PM}$ at zero and finite temperature, Phys. Rev. B 93, 104411 (2016).

[45] B. M. Huddart, M. Gomilšek, T. J. Hicken, F. L. Pratt, S. J. Blundell, P. A. Goddard, S. J. Kaech, J. L. Manson, and T. Lancaster, Magnetic order and ballistic spin transport in a sineGordon spin chain [dataset] (2021), doi:10.15128/r2bv73c042s.

[46] J. P. Perdew, K. Burke, and M. Ernzerhof, Generalized Gradient Approximation Made Simple, Phys. Rev. Lett. 77, 3865 (1996).

[47] J. H. Brewer, S. R. Kreitzman, D. R. Noakes, E. J. Ansaldo, D. R. Harshman, and R. Keitel, Observation of muon-fluorine "hydrogen bonding" in ionic crystals, Phys. Rev. B 33, 7813 (1986).

[48] T. Lancaster, S. J. Blundell, P. J. Baker, M. L. Brooks, W. Hayes, F. L. Pratt, J. L. Manson, M. M. Conner, and J. A. Schlueter, Muon-Fluorine Entangled States in Molecular Magnets, Phys. Rev. Lett. 99, 267601 (2007). 